EPiC Series in Engineering
Volume 3, 2018, Pages 282-289
HIC 2018. 13th International
Conference on Hydroinformatics

\title{
Optimal Sensor Placement and leak/burst localisation in a water distribution system using spatially-constrained inverse-distance weighted interpolation
}

\author{
Shaun Boatwright ${ }^{1,2}$, Michele Romano ${ }^{2}$, Stephen Mounce $^{1}$, Kevin \\ Woodward $^{2}$ and Joby Boxall ${ }^{1}$ \\ ${ }^{1}$ Pennine Water Group, Department of Civil and Structural Engineering, University of Sheffield, \\ Sheffield, S1 3JD, UK. \\ ${ }^{2}$ United Utilities, Lingley Mere Business Park, Warrington, WA5 3LP, UK. \\ mea08srbesheffield.ac.uk
}

\begin{abstract}
Water loss from water distribution systems (WDS) is an ongoing problem which poses a significant risk to water resources around the world. This paper presents a novel combined sensor placement - leak/burst localisation methodology which forms, and analyses by using sc inverse-distance weighted (IDW) interpolation, a sensitivity matrix to determine, on average, how accurately each sensor configuration localises leaks/bursts modelled at all nodes in a WDS. For a given number of sensors, the multi-objective evolutionary algorithm determines the optimal location of sensors to maximise the leak/burst localisation performance using the sc-IDW outputs in its objective function. Once the optimal sensor location is selected, the sc-IDW technique is used when new leaks/bursts occur in the WDS to determine their approximate location. A benchmark WDS was used to compare the leak/burst localisation performance against a baseline sensor placement technique. The comparison indicated that by using the sc-IDW technique for both the sensor placement and leak/burst localisation steps the leak/burst search area was reduced in size by between 9 and $26 \%$. Reducing the leak/burst search area allows field teams to more quickly repair a leak/burst and reduce the impact that it has on water company operational efficiency and customer service.
\end{abstract}

\section{Introduction}

Water companies around the world must manage large, complex, underground Water Distribution Systems (WDS) whilst providing high levels of service on limited budgets. The size and complexity of 
most WDS means that problems are inevitable. One of the most difficult problems faced by water companies is managing the loss of water from WDSs. Water loss, caused by failure of pipes within the WDS, can impact upon customer service levels and operational efficiency by causing supply interruptions. This loss of treated and frequently pumped water (along with associated costs) causes it to remain the target of considerable concern for water companies and the public alike. Due to the inevitability of the problem, water companies must devise strategies to minimise the consequences of these problems, once they have arisen, and to ensure that normal operation is restored as quickly as possible. The consequences of a leak/burst are highly dependent upon the length of time between its initiation and repair (its "lifecycle" - [1]). Therefore, to reduce the consequences associated with a leak/burst it is desirable to quickly determine whether a leak/burst is present or not (i.e. detecting it) and identify its approximate location (i.e. localising it). This means that field teams deployed to find the leak/burst only have to search a small area in the WDS and can repair the leak/burst quickly.

In the UK, and many other countries, WDS are divided into sectors called District Metered Areas (DMAs) to allow monitoring of both flow and pressure in each sector. Techniques for analysing DMA flow and pressure data in order to detect [2] and locate [3] leaks/bursts, by identifying when and where changes indicative of a leak/burst have occurred, are available and have been applied to real WDS. The success of these methods (especially for localisation) is limited by the number of sensors which can be placed in each DMA (and therefore the entire WDS), and as with current technology, there is a tradeoff between the improvements in performance resulting from installing more sensors and the cost of installing and maintaining them. To minimise these costs, selecting the optimal number and location of sensors in a DMA is a crucial, but often overlooked, step. Even when one of the available sensor placement algorithms (for example - [4]) is used, usually the sensor locations selected are not optimised for the specific localisation technique being used, which represents a significant potential opportunity for improvement.

Geostatistical techniques for the localisation of leaks/bursts have been proposed [3] due to their ability to infer the value of a variable at locations which are not measured by using available measurements and, hence, to enable higher leak/burst localisation performance to be achieved for a given number of sensors. Those geostatistical techniques, however, used Euclidean distance between the deployed sensors, therefore neglecting the network layout of a DMA. To overcome this limitation, the authors proposed a sc-IDW interpolation technique [5] that used the actual distance along pipes and the connectivity of the WDS to determine the approximate location of a new leak/burst.

In view of the above, to overcome the previously described sensor placement limitation, a novel sensor placement algorithm (based upon the sc-IDW technique) is proposed in this paper. This novel combined sensor placement - leak/burst localisation methodology aims at ensuring that optimal sensor locations (with respect to the leak/burst localisation technique) are selected.

\section{Methodology}

\subsection{Overview}

The novel combined sensor placement - leak/burst localisation methodology aims to determine the approximate location of a new leak/burst in a DMA by analysing the data from a number of installed pressure sensors. Once the desired number of sensors has been selected, the methodology uses a MultiObjective Evolutionary Algorithm (MOEA) to calculate the optimal location of pressure sensors in the DMA. This is achieved by hydraulic modelling of leaks/bursts at all nodes in the DMA and evaluating (using a sc-IDW objective function) the localisation performance of each configuration of sensors for every leak/burst modelled. After the optimal sensor configuration has been determined, the sc-IDW technique is used again to calculate the approximate location of a new leak/burst in a DMA (once a 
leak/burst has been identified or is suspected). The remainder of this section will describe (i) the scIDW technique, which is used by both (ii) the optimal sensor placement procedure (iii) and the leak/burst localisation technique.

\subsection{Spatially constrained inverse-distance weighted interpolation}

The core of the sensor placement/leak localisation methodology is the sc-IDW technique. Traditional inverse-distance weighted (IDW) interpolation [6] is a geostatistical technique which allows estimation of a variable at a point which is not measured using a series of measurements at other points. The value of the variable at each unmeasured location is based purely upon the distance of the unmeasured location from each of the measured locations and can be described mathematically using Equation 1.

$$
Z_{e s t, j}=\sum \frac{z_{\mathrm{i}}}{\left[d_{i j}+s\right]^{p}} / \sum \frac{z_{\mathrm{i}}}{[1+s]^{p}}
$$

where: $Z_{\text {est }, j}=$ estimated value at location $j ; z_{i}=$ value at measured location $i ; d_{i j}=$ distance between $i$ and $j ; s=$ smoothing factor; and $p=$ weighting power. As was discussed previously by the authors [5, 7] and due to the geometry/connectivity of WDS, the selection of an appropriate distance function (i.e. to be used in place of $d_{i j}$ in Equation 1.) for the leak/burst localisation problem is of critical importance. The authors previously developed a distance function which calculates the distance between points in the WDS by considering the distance travelled along pipes and used this to perform the sc-IDW interpolation. This distance function forms the core of the sc-IDW technique used here.

\subsection{Optimal sensor placement procedure}

The optimal placement of sensors seeks to maximise the localisation performance for a given number of sensors. It comprises several stages including: (i) formation of the sensitivity matrix by hydraulic modelling of leaks/bursts at all nodes (ii) evaluating each sensor configuration using the scIDW objective function (with pipe length as the distance function) to calculate the average localisation performance for all the modelled leaks/bursts and (iii) selecting the optimal configuration of sensors using a MOEA.

The first step in the optimal sensor placement procedure is to form the sensitivity matrix [8] by hydraulic modelling of leaks/bursts at every node, in turn, and recording the resulting change in pressure at every node which occurs due to each individual leak/burst. The change in pressure is then calculated as a function of the normal pressure at node $i\left(p_{i}\right)$ and the pressure at node $i$ due to a leak at node $j\left(p_{i j}\right)$ using the chi-squared $\left(\chi^{2}\right)$ error described by Equation 2.

$$
\chi_{i j}^{2}=\frac{\left(p_{i j}-p_{i}\right)^{2}}{p_{i}}
$$

The calculated chi-squared values are stored in an $n$ by $n$ sensitivity matrix (where $n$ is the number of nodes in the hydraulic model being analysed). The chi-squared value is a measure of change between the leak condition and normal condition (with no leaks/bursts present) and it is used, as a pressure residual measure, in place of the absolute difference $\left(p_{i j}-p_{i}\right)$ because it accounts for the expected (or normal) value of pressure at each node and therefore enables a fair comparison between nodes at different pressures to take place [9]. Equation 2. describes the chi-squared error for a single period simulation (as per the examples included in the case study presented here) but this can be easily adapted for extended period simulations by summing over multiple time steps. Leaks/bursts were modelled as emitters using Equation 3.

$$
q=C p^{\gamma}
$$

where: $q$ is the flow rate through the emitter, $p$ is the pressure at the node where the leak is being modelled, $C$ is the discharge coefficient and $\gamma$ is the fixed pressure exponent. To perform the optimal sensor placement procedure the GALAXY MOEA [10] is used. The GALAXY MOEA has been used 
previously for a number of optimisation problems related to WDS design and maintenance. A wellknown issue with evolutionary algorithms is the large number of parameters which usually need to be fine-tuned to ensure that they perform well (in terms of finding suitable solutions in a timely manner). To initialise the GALAXY MOEA, however, the user must specify only two MOEA parameters; the number of function evaluations (NFEs) and the desired size of the starting population. In the work presented here, the user must also select the desired number of sensors which are to be deployed in the network being analysed. The GALAXY MOEA generates a number of candidate solutions, equal to the size of the starting population, randomly. Each candidate solution is an $m$ by 1 vector (where $m$ is the desired number of sensors) containing the indices of the hydraulic model nodes which have been selected for sensor installation. The candidate solutions are evaluated, in turn, using the sc-IDW objective function. The sc-IDW objective function extracts the columns from the sensitivity matrix relating to the sensors in the candidate solution being evaluated (i.e. the pressure residuals for all leaks/bursts at the nodes contained in the candidate solutions) and, for each leak/burst runs the sc-IDW technique to estimate the value of pressure residual for every node to create the sc-IDW sensitivity matrix. In order to determine the value of the sc-IDW objective function for each candidate solution it is necessary to determine which nodes should be included in the leak/burst search area for each leak/burst and, therefore, how large the total leak/burst search area was across all of the modelled leaks/bursts. To do this a sc-IDW threshold is introduced (see Case Study section for details). For each modelled leak/burst (row of the sc-IDW sensitivity matrix) the sc-IDW threshold distinguishes between the leak/burst search area and the rest of the nodes. Nodes with an estimated pressure residual greater than or equal to the sc-IDW threshold for a given leak/burst are classed as part of the leak/burst search area. The sc-IDW objective function then determines for all leaks/bursts whether the sc-IDW localisation was successful (i.e. node burst $_{\in} \in$ nodes $_{\text {search area }}$ ). If a leak/burst was correctly located then the sc-IDW counts the number of nodes in the leak/burst search area. Conversely, for each leak/burst which was not correctly located (i.e. node burst $\notin$ nodes $_{\text {search area }}$ ) all nodes for that leak/burst are counted, as per Equation 4. This ensures that the sc-IDW objective function favours (and gives a lower value to) candidate solutions which correctly locate a higher number of leaks/bursts. The value of objective function for a candidate solution is then the total of the calculated objectives for all individual leaks/bursts, as described by Equation 5.

$$
\begin{aligned}
& \text { objective }_{\text {burst }}= \begin{cases}\sum \text { nodes }, & \text { if node } \\
\text { burst } & \notin \text { nodes }_{\text {search area }} \\
\sum \text { nodes }_{\text {search area }}, & \text { if node } \text { burst }_{\text {bition }} \in \text { nodes }_{\text {search area }}\end{cases} \\
& \text { objective }_{\text {candidate solution }}=\sum_{i=1}^{n} \text { objective }_{\text {burst }}
\end{aligned}
$$

Once every member of the starting population has been evaluated using the sc-IDW objective function, the solutions are ranked from best (the solution(s) with the lowest value of sc-IDW objective function) to worst (the solution(s) with the highest value of sc-IDW objective function) by the GALAXY MOEA. The best solutions are retained and the worst are eliminated. Those solutions not falling in the "worst" or "best" categories are modified slightly by changing some of the proposed candidate solutions (so that the sensors are installed in different locations) and are then included in the next generation. All retained solutions then form the basis of the new population, which is the same size as the starting population. The evaluation and selection of new populations is repeated a number of times, until the user specified NFEs have been completed and the optimal sensor configuration has been determined for the specified number of sensors to be deployed. The procedure can be repeated for as many scenarios (i.e. number of sensors to be deployed in the WDS being analysed) as required prior to selecting the desired, final sensor configuration. For further information with regard to the initialisation, mutation, ranking and selection of the candidate solutions the reader is referred to [10].

\subsection{Leak/burst localisation technique}

Once the final sensor configuration has been selected from the available optimal configurations and a new leak/burst has occurred in the WDS under consideration the sc-IDW leak/burst localisation 
technique analyses the incoming pressure signals and estimates the pressure residuals for all nodes in the WDS based upon those calculated for each of the installed sensors. As described in section 2.3 the sc-IDW threshold is used to determine which nodes contain the likely location of the leak/burst which can then be passed onto field teams for further investigation.

\section{Case Study}

\subsection{Description and overall results}

To demonstrate its performance, the proposed novel combined sensor placement - leak/burst localisation methodology was used to determine the optimal sensor configurations (using the optimal sensor placement procedure) and, subsequently, localise (using the sc-IDW based leak/burst localisation technique) the leaks/bursts modelled at all nodes using an offline hydraulic model for the Bakryan benchmark WDS [11]. For comparison, a sensor placement technique has been chosen from the literature [12] (referred to as the baseline sensor placement technique) and has been used to also determine optimal sensor configurations. Two sc-IDW thresholds were used, in combination with each sensor placement technique, to examine how the sc-IDW threshold affects the optimal logger configurations and the leak/burst localisation performance. The first sc-IDW threshold (the global mean) was calculated as the mean of the entire sc-IDW sensitivity matrix (similar to [12]) and the second (the local mean) was calculated as the mean of the estimated pressure residuals for a particular leak/burst (using a single row of the sc-IDW sensitivity matrix). Only optimal sensor configurations with between two and four sensors (inclusive) have been considered here due to the size of the Bakryan benchmark WDS, shown in Figure 1., which contains 35 nodes and 58 pipes (ranging in size from 100 to $900 \mathrm{~mm}$ ) totalling $102 \mathrm{~km}$. The Bakryan benchmark WDS was selected from the literature primarily due to its geometry and highly connected structure. All leaks/bursts were modelled using an emitter coefficient $(C)$ of 3 and a fixed pressure exponent $(\gamma)$ of 0.5 (to reduce the size of the solution space) in a single period simulation. Once the optimal sensor configurations were determined, the sc-IDW based leak/burst localisation procedure was performed for all leaks/bursts.
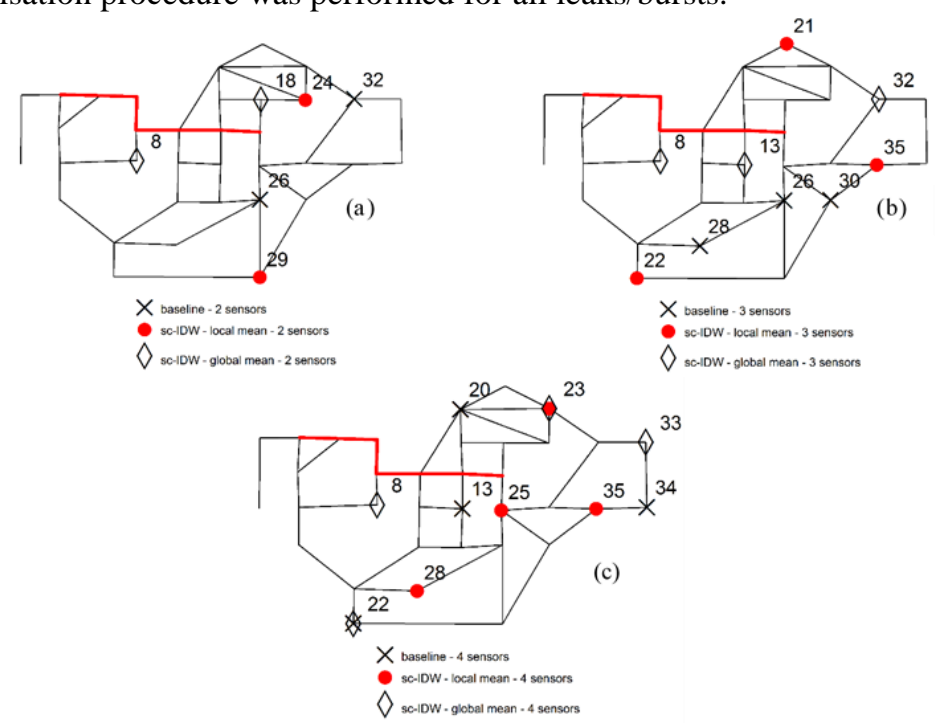

Figure 1: The Bakryan benchmark WDS with optimal sensor configurations determined by the sc-IDW optimal placement technique with local mean (circles), global mean (diamonds) and the baseline sensor placement technique (crosses) with (a) 2 sensors (b) 3 sensors (c) 4 sensors. Bold lines denote pipes which are duplicated 
For each sensor placement technique the value of the sc-IDW objective function (calculated as described in section 2.3), the number of leaks/bursts correctly located and the indices of the nodes in the optimal sensor configuration have been calculated and are shown in Table 1. below. The value of the sc-IDW objective function, is significantly lower using the optimal sensor placement procedure than for the baseline sensor placement technique (for the same number of sensors and sc-IDW threshold).

\begin{tabular}{|c|c|c|c|c|c|}
\hline $\begin{array}{c}\text { Sensor } \\
\text { placement }\end{array}$ & $\begin{array}{l}\text { sc-IDW } \\
\text { threshold }\end{array}$ & $\begin{array}{c}\text { Number } \\
\text { of } \\
\text { sensors }\end{array}$ & $\begin{array}{l}\text { Node indices of } \\
\text { optimal sensor } \\
\text { locations }\end{array}$ & $\begin{array}{c}\text { sc-IDW } \\
\text { objective } \\
\text { function } \\
\text { value }\end{array}$ & $\begin{array}{c}\text { Total } \\
\text { leaks/bursts } \\
\text { correctly } \\
\text { located } \\
\end{array}$ \\
\hline \multirow{3}{*}{$\begin{array}{l}\text { Optimal (sc- } \\
\text { IDW) }\end{array}$} & \multirow{3}{*}{ Local Mean } & 2 & 24,29 & 643 & 34 \\
\hline & & 3 & $21,22,35$ & 591 & 34 \\
\hline & & 4 & $23,25,28,35$ & 590 & 31 \\
\hline \multirow{3}{*}{$\begin{array}{l}\text { Optimal (sc- } \\
\text { IDW) }\end{array}$} & \multirow{3}{*}{$\begin{array}{l}\text { Global } \\
\text { Mean }\end{array}$} & 2 & 8,18 & 895 & 12 \\
\hline & & 3 & $8,13,32$ & 834 & 24 \\
\hline & & 4 & $8,22,23,33$ & 831 & 25 \\
\hline \multirow{3}{*}{ Baseline [12] } & \multirow{3}{*}{ Local Mean } & 2 & 26,32 & 811 & 25 \\
\hline & & 3 & $28,30,32$ & 742 & 26 \\
\hline & & 4 & $13,20,22,34$ & 649 & 30 \\
\hline \multirow{3}{*}{ Baseline [12] } & \multirow{3}{*}{$\begin{array}{l}\text { Global } \\
\text { Mean }\end{array}$} & 2 & 26,32 & 1157 & 6 \\
\hline & & 3 & $28,30,32$ & 1121 & 7 \\
\hline & & 4 & $13,20,22,34$ & 999 & 20 \\
\hline
\end{tabular}

Table 1: Comparison of the performance of the optimal sensor placement procedure against the baseline sensor placement technique using both the local and global mean sc-IDW thresholds

This is because the average leak/burst search area produced for each combination of leak/burst location and sensor configuration is accounted for by the sc-IDW objective function of the GALAXY MOEA, unlike other sensor placement techniques found in the literature. It is clear from Table 1. that there is not a precise relationship between the value of the sc-IDW objective function and the number of leaks/bursts which are correctly located for each of the optimal sensor configurations, although generally a proportional relationship can be seen. This is because the sc-IDW objective function also has to factor in the size of the search area and trade this off against the number of leaks/bursts localised correctly (because increasing the search area will lead to a higher rate of correct leak/burst localisations).

It is clear from Table 1. that the combination of the optimal sensor placement procedure and the local mean sc-IDW threshold leads to the best localisation performance for each number of sensors. This is intuitive for two reasons. Firstly, the sensors are placed optimally with respect to the sc-IDW leak/burst localisation technique and secondly, the local threshold is calculated based upon the estimated pressure residuals for a given leak/burst location only. The global mean however is calculated based upon the estimated pressure residuals of all leak/burst locations, which have a much greater range and hence, is almost arbitrary when used to determine the leak/burst search area for a single leak/burst. For all combinations of sensor placement technique and sc-IDW threshold used, increasing the number of sensors led to a decrease in the value of the sc-IDW objective function. This is to be expected and, 
therefore, shows the suitability of the sc-IDW objective function within the optimal sensor placement procedure.

\subsection{Localisation example}

To further demonstrate the leak/burst localisation performance of each combination of sensor placement technique and sc-IDW threshold an example leak/burst has been chosen and the sc-IDW leak localisation technique has been run to determine the leak/burst search area for each. The example leak/burst was modelled at node 24 in the Bakryan benchmark WDS, and the resulting leak/burst search areas produced for the scenario with four pressure sensors are shown in Figure 2.

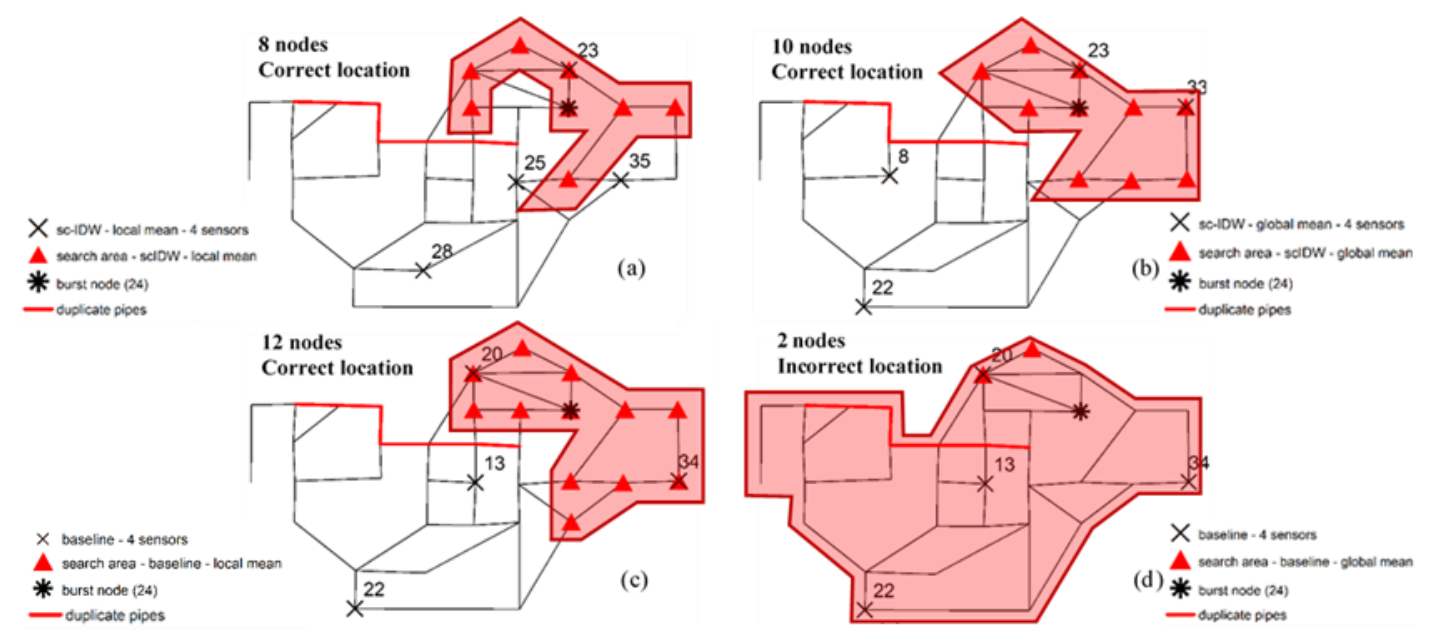

Figure 2: Leak/burst search areas (shaded boxes) including the search nodes (triangles) and optimal sensor locations (crosses) calculated by sc-IDW leak localisation technique using the optimal sensor placement technique with (a) local mean and (b) global mean and the baseline sensor placement technique with (c) local mean and (d) global mean for a leak/burst modelled at node 24 (asterisk)

It can be seen for the example leak/burst that the optimal sensor placement with the local mean scIDW threshold produces the smallest leak/burst search area (requiring that an area covering eight nodes would need to be searched). Both leak/burst search areas produced using the local mean sc-IDW threshold are smaller than for their global mean counterparts. Figure 2(d) demonstrates the problem with the global mean, which was highlighted previously. In this case the leak/burst was incorrectly located because the global mean has not been calculated with respect to the pressure residuals caused by it and two nodes have been selected due to their proximity to the pressure sensor deployed at node 20 (nodes 20 and 21 form the search area).

\section{Conclusions and Future Work}

Presented here is not only a methodology for localising leaks/bursts and optimally placing sensors but also a general methodology, which can be extended to other data-driven leak/burst localisation methodologies, which rely on placing additional sensors in a WDS. The proposed novel combined sensor placement - leak/burst localisation methodology and a baseline sensor placement technique were applied to the Bakryan benchmark WDS, in combination with two thresholds, to determine the optimal sensor configurations. Using these optimal sensor configurations leaks/bursts modelled at all nodes in the Bakryan benchmark WDS were subsequently localised. The results of the localisation included in 
the case study showed that, on average, the sc-IDW leak/burst localisation technique in combination with a local mean threshold (calculated for each individual leak/burst) resulted in the best localisation performance. An example leak/burst modelled at node 24 further demonstrated the effectiveness of the sc-IDW leak/burst localisation technique. The novel combined sensor placement - leak/burst localisation methodology can therefore enable significant reduction in the leak/burst search area and, hence, the time taken for field teams to find a leak/burst in a WDS. This offers the potential to improve customer service and increase operational efficiency by minimising the negative consequences of leaks/bursts. As part of the case study included here, the sc-IDW parameters (i.e sc-IDW weighting exponent $(p)$ and threshold) were fixed in order to reduce the size of the solution space but are, in fact, critical to the leak/burst localisation performance of the novel combined sensor placement - leak/burst localisation methodology. Future work to incorporate these into the GALAXY MOEA optimisation will ensure that the optimal combination of sensor configurations and sc-IDW parameters are determined, to maximise the leak/burst localisation performance. This will also ensure that the sc-IDW parameters can be tailored for individual WDS and updated as the boundary conditions of the WDS change over time (due to operational changes or deterioration in condition). Further development and testing of the novel combined sensor placement - leak/burst localisation methodology, to include extended period simulations and more realistic approaches to leak/burst hydraulic modelling (such as pressure dependent demand hydraulic modelling [13]) using hydraulic models of "real" WDS, is required prior to its operational use.

\section{References}

[1] S.R. Mounce, J. Machell, Burst detection using hydraulic data from water distribution systems with artificial neural networks, Urban Water J. 3 (1) (2006) 21-31.

[2] M. Romano, Z. Kapelan, D. A. Savic, Automated detection of pipe bursts and other events in water distribution systems, J. Water Resour. Plan. Manage. 140 (4) (2014) 457-67.

[3] M. Romano, Z. Kapelan, D. A. Savic, Geostatistical techniques for approximate location of pipe burst events in water distribution systems, J. Hydroinform., 15 (3) (2013) 634-51.

[4] J. Blesa, F. Nejjari, R. Sarrate, Robust sensor placement for leak location: Analysis and design, J. Hydroinform., 18 (1) (2016) 136-48.

[5] S. Boatwright, M. Romano, S. R. Mounce, K. Woodward, J. B. Boxall, Approximate location of leaks and bursts in a district metered area using statistical process control and geostatistical techniques, Proc. Computing and Control for the Water Industry Conference, Amsterdam, Netherlands (2016).

[6] D. Zimmerman, C. Pelvic, A. Ruggles, M. P. Armstrong, An experimental comparison of ordinary and universal Kriging and inverse distance weighting, Math. Geol., 31 (4) (1999) 375-90.

[7] S. Boatwright, M. Romano, S. R. Mounce, K. Woodward, J. B. Boxall, Investigating a spatially constrained geostatistical technique for approximate leak/burst event location, Proc. Computing and Control for the Water Industry Conference, Sheffield, UK (2017).

[8] R. Pudar and J. Liggett, Leaks in pipe networks, J. Hydraul. Eng., 118 (7) (1992) 1031-46.

[9] B. Farley, S. R. Mounce, J. B. Boxall, Field testing of an optimal sensor placement methodology for event detection in an urban water distribution network, Urban Water J., 7 (6) (2010) 345-56.

[10] Q. Wang, D. A. Savic, Z. Kapelan, GALAXY: A new hybrid MOEA for the optimal design of Water Distribution systems, Water Resour. Res., 53 (2017) 1997-2015.

[11] S. Lee, S. Lee, Genetic algorithms for optimal augmentation of water distribution networks. J. Korea Water Resour. Assoc., 34 (5) (2001) 567-75.

[12] B. Farley, Optimal sensor placement for detection and location of leak/burst events, PhD thesis, University of Sheffield, Sheffield, UK (2010).

[13] O. Giustolisi, D. A. Savic, Z. Kapelan, Pressure-driven demand and leakage simulation for water distribution networks. J. Hydraul. Eng., 134 (5) (2008) 626-35. 